conference at Exeter last week on the teaching of mathematics to physicists, held under the auspices of the Institutes of Physics and Mathematics. Professor H. B. Griffiths (Southampton) set the ball rolling with a description of the search for order in various categories of objects and manoeuvres which made many feel from the start that the similarity between the two disciplines was indeed considerable.

Mr. Cyril Hope, Director of the Midlands Mathematics Experiment, gave an idea of what is likely to be expected at A-level and beyond from the changes due to the School Mathematics Project and his own. Few will regret the passing of geometrical optics, many will welcome an appreciation among schoolchildren of "acceptable accuracies", some will question the introduction of probability and statistics, but all will be grateful at the attempt to get teachers from out of the rut into which examinations have so often led them. Just as science teachers have said for years, so mathematicians put much of the blame for the quality and character of the teaching on examiners.

At Exeter, Sir Nevill Mott, speaking on the role of mathematics in physics, said that the relationship between applied mathematics and physics could be expected to raise its ugly head, but only one voice was raised against the notion that in school mathematics, dynamics must and, indeed, can only arise logically from experiment. The introduction of trolleys and ticker tape in the courses of the Nuffield Science Teaching Project has seemingly completely changed the understanding of kinematics.

Dr. S. Raines (Imperial College), speaking on undergraduate mathematics, said that the Peierls report published last year showed the very wide range of time spent on mathematics in university departments of physics. The frequently uneasy relationship between the teachers of the two disciplines is often blamed not on the content of what the mathematicians teach to physics students but on their approach and the lack of illustrations and relevance to the physical world. Dr. Raines also spoke of the inadequate preparation in the schools for what is required by a new undergraduate-a point disputed by Dr. Bryan Thwaites, who made a special plea for a single "core" subject of mathematics with a wide variety of additional options at A-level. The sixth form is crucial to the British tertiary system of education, and the mathematicians must satisfy the prospective physicists, chemists, engineers and mathematicians as well as the biologists and sociologists. Dr. Thwaites said that it was essential and urgent that the Department of Education and Science should give a lead to curriculum reformers in support of the A-level examination as a school-leaving examination.

At the end of their successful meeting, the two institutes agreed to establish a joint working party on the teaching of mathematics to physicists. The objective is to design an improvement of the sixth-form mathematics curriculum, and to collaborate with the Schools Council.

\section{Structure and Properties of Liquids}

Professor H. N. V. Temperley writes:

The Faraday Society held a discussion with the above title from April 11-13 at the University of Exeter. Growing interest in the subject was reflected in the attendance of more than 280 , ranging from pure mathematicians and chemists to electrical engineers. (For a survey, see Nature, 211, 906; 1966.)

The section on equilibrium theory was introduced by the Spiers memorial lecture given by Professor G. S. Rushbrooke (Newcastle). It is interesting that the date of the last Faraday Society discussion on liquids (1936) corresponded with the beginning of the modern approach to liquids by means of the twomolecule distribution function. The appropriate mathematical tools for studying this are integral equations, series expansions or some combination of the two. All workable integral equations are necessarily approximate and it is still far from clear why the newer ones (Percus-Yevick and hypernetted chain) work so much better than the older ones (Kirkwood and Born-Green). As between the two newer ones, the Percus-Yevick equation is very satisfactory at high densities and temperatures, but Henderson (University of Waterloo) provided some evidence that the hypernetted chain equation works better at lower temperatures. No known equation gives satisfactory results in the critical region.

The studies initiated by Bernal of the geometry of a random arrangement of spheres have been very fruitful. Besides the latest developments of this work from Finney (Birkbeck), we heard of various attempts to simulate a heap by computer methods. A pleasing film showed an assembly of disks gradually swelling and forcing one another into an ordered configuration. Despite some encouraging indications, it is still not known whether the integral equation approach gives a proper description of the solidification transition.

There was a lively discussion about the properties of water and hydrogen-bonded liquids. Even with the much better X-ray techniques now available, and with the help of infra-red spectroscopy, it is even now only possible to put forward tentative structures.

A survey by Egelstaff (U.K. Atomic Energy Research Establishment, Harwell) called attention to the various types of information obtainable from neutron X-ray and optical scattering. In contrast with the $\mathrm{X}$-ray work, it is possible to study the correlation between two molecules in time as well as distance, which is important from the point of view of transport properties. As possible further generalizations one can study correlation as a function of angle (Buckingham; Bristol) and of frequency (Stecki; Polish Academy of Sciences). Both of these approaches were shown to be potentially fruitful. Nuclear magnetic resonance and nuclear relaxation (Pople, Dweck and Richards) in which the nucleus acts as a probe are also useful in some liquids.

A typical example of possible cancellation of errors is provided by attempts to represent the interaction of molecules by the simple Lennard-Jones $6-12$ potential function. This seems to work better for liquid argon than it should on the basis of evidence from the gas and the solid. It may be that three-molecule interactions are tending to cancel out the fact that the 'bowl' of the Lennard-Jones function is almost certainly too wide and too shallow.

By way of a look into the future, Prings (California Institute of Technology) thought that it should soon be possible to determine an inter-molecular interaction function directly from X-ray scattering. 